\title{
Nitric Oxide Scavenger Carboxy-PTIO Impaired Memory of Avoidance Conditioning in Goldfish
}

\author{
X. Xu, K. Zmolek \\ Department of Psychology, Grand Valley State University, Allendale, MI 49401, USA \\ Email: xux@gvsu.edu
}

Received January 2014

\begin{abstract}
The long-term potentiation (LTP), an activity-induced increase in the efficacy of neurotransmission, has long been conceived to be a physiological correlate of learning and memory. Investigations of synaptic transmission suggest that the postsynaptic N-methyl-D-aspartate (NMDA) receptor is the upstream mediator of LTP, while nitric oxide (NO), a retrograde messenger from postsynaptic neurons to presynaptic neuron, is the downstream mediator of LTP. Our previous studies showed that microinjections of NMDA receptor antagonist D-AP5 to the goldfish telencephalon prior to trainings impaired learning of avoidance conditioning in goldfish. However, microinjections of D-AP5 to the goldfish telencephalon immediately following trainings did not impair memory of avoidance conditioning. Carboxy-PTIO is a NO scavenger that prevents NO from reaching the presynaptic neurons. The present study investigated the effects of microinjections of carboxyPTIO to the goldfish telencephalon immediately following trainings on avoidance conditioning. The results showed that microinjections of carboxy-PTIO to the goldfish telencephalon immediately following trainings impaired memory of avoidance conditioning.
\end{abstract}

\section{Keywords}

Avoidance Conditioning, Memory Consolidation, Nitric oxide, Goldfish

\section{Introduction}

Several neurochemical steps of long-term potentiation (LTP), an activity-induced increase in the efficacy of neurotransmission, have been identified. Investigations of synaptic transmission show that the neurochemical steps involved in the LTP initially require activation of postsynaptic receptors known as N-methyl-D-aspartate (NMDA) receptors [1]. Activation of NMDA receptors leads to the influx of $\mathrm{Ca}^{2+}$ into postsynaptic neurons [2]. The influx of $\mathrm{Ca}^{2+}$ into postsynaptic neurons activates the nitric oxide synthase (NOS) enzyme that leads to the synthesis of nitric oxide (NO), a retrograde messenger carrying signals backward from postsynaptic to presynaptic neurons [3]. NO then triggers further biochemical reactions that lead to LTP [4]. Thus, the NMDA receptor is the upstream mediator of LTP, while NO is the downstream mediator of LTP [5]. 
LTP has long been conceived to be a physiological correlate of learning and memory [6]. Our previous studies showed that NMDA receptor antagonists impaired learning when given before training, but did not impair memory when given after training [7] [8]. Furthermore, microinjections of NMDA receptor antagonist D-AP5 to the goldfish telencephalon prior to trainings impaired learning of active avoidance conditioning through its interaction with telencephalic NMDA receptors in goldfish [9]. However, microinjections of D-AP5 to the goldfish telencephalon immediately following trainings did not impair memory of avoidance conditioning [9] CarboxyPTIO is a NO scavenger that prevents NO from reaching the presynaptic neurons. The present study investigated the effects of microinjections of carboxy-PTIO to the goldfish telencephalon immediately following trainings on active avoidance conditioning.

\section{Methods}

\subsection{Subjects and Experimental Drug}

Goldfish (Carassius auratus L.), which were 8 - $10 \mathrm{~cm}$ in length and obtained from local pet stores, were kept in large tanks for several weeks prior to experiments. During experiments, fish were kept in individual compartments of partitioned tanks at $20^{\circ} \mathrm{C} \pm 1{ }^{\circ} \mathrm{C}$ with a $12 \mathrm{~h}$ light-dark cycle (0700-1900 light). Experiments were conducted during the light cycle. 2-(4-carboxyphenyl)-4,4,5,5-tetramethylimidazoline-1-oxyl-3-oxide potassium salt (carboxy-PTIO) (Sigma-Aldrich, St. Louis, MO, USA) was dissolved in saline.

\subsection{Microinjection}

The goldfish telencephalon is located at the anterior end of the head and right beneath the skull. The skull of the goldfish has characteristic sutures that are visible without removing any tissue. Because the skull of the goldfish used in the study was easy to penetrate with a needle without harming the goldfish, a 26-gauge needle with a rubber stopper making the length of needle $2 \mathrm{~mm}$ was used to make needle holes in the skull relative to the sutures. The punctures were made on line with the posterior margins of the iris of the eyes and $0.5 \mathrm{~mm}$ lateral to the midline bilaterally. A $10 \mu \mathrm{l}$ Hamilton syringe with a $7 \mathrm{~mm}$ length needle was used to make injections through the punctures at $7 \mathrm{~mm}$ ventral to the top of the skull. The injection was $0.5 \mu \mathrm{l}$ per side and $1 \mu \mathrm{l}$ total per fish. Repeated injections were made through the same punctures and at the same depth, therefore to the same location in the brain. The accuracy of the placement of the microinjection was determined by injecting methylene blue dye through the same punctures and at the same depth at the end of testing. Fish were sacrificed with an overdose of tricaine and the skull of fish was then removed to verify the location of microinjections. Fish in which both sides of telencephalon were dyed blue were retained in the study.

\subsection{Apparatus}

Fish were trained and tested individually in three identical fish shuttle-boxes connected to a Smart Control (Med Associates Inc., Albans, Vermont). The fish shuttle-box consisted of a water-filled tank $(41 \mathrm{~cm}$ in length $\times 20.5$ $\mathrm{cm}$ in width $\times 10.5 \mathrm{~cm}$ in height) separated by an opaque barrier $(20.5 \mathrm{~cm}$ in width $\times 10.5 \mathrm{~cm}$ in height $)$ into two equal compartments. A rectangular opening $(8 \mathrm{~cm}$ in width $\times 2.5 \mathrm{~cm}$ in height) in the barrier allowed fish to swim freely from one side of the tank to the other. The crossing movement of the fish was monitored by four infrared light beams and their corresponding detectors located on the long sides of the tank. There was a light $(75 \mathrm{~W}, 125 \mathrm{~V}, \mathrm{DC})$ at each end of the tank and there were two stainless steel electrode plates $(18 \mathrm{~cm}$ in length $\times$ $19.5 \mathrm{~cm}$ in width) at the top and bottom of each compartment. The water in fish shuttle-boxes was completely changed between fish.

\subsection{Active Avoidance Paradigm}

Fish were placed in the shuttle-boxes for $5 \mathrm{~min}$, and then a trial began with the onset of the light on the side of the fish's location. After the light was on for 20 seconds, a repetitive mild electrical shock $(1 \mathrm{~V} / \mathrm{cm} \mathrm{DC}$, pulsed $200 \mathrm{~ms}$ on and $800 \mathrm{~ms}$ off) was administered, along with the light, for 20 seconds through the water by means of electrodes. At the end of 40 seconds or upon a crossing response by fish during the 40 seconds, both the light and electrical shock was switched off and the trial ended. After an intertrial interval (ITI) ranging from 25 - 55 seconds, another trial began. 
Fish initially swam through the opening only after receiving several shocks. The crossing response following the onset of both light signal and electrical shock to escape the electrical bodyshock is defined as an escape response. During the training sessions, fish gradually learned to swim from the lighted end to the dark end to avoid an electric bodyshock. The crossing response following the onset of the light signal but before the onset of electrical shock to avoid the electrical bodyshock is defined as an avoidance response. Fish were trained semiweekly on Experimental Days 1, 4, and 8. On Experimental Day 11, fish were tested for retention of learning. A training session consisted of 20 trials, and the testing session consisted of 10 trials. The measurements were the number of avoidances, escapes and crossings during ITI; and the measurements were then converted into percentages. To reduce the rate of crossing during ITI, crossing during ITI was immediately followed by a single bodyshock. All experiments were fully automated through the Smart Control and a single 486 computer that programmed stimuli, monitored and recorded behavior of fish. Fish that showed ITI crossing $30 \%$ or less during training and testing sessions were retained in the study. Percentage of avoidance responses during the test session was used as an indicator of retention.

\subsection{Procedure}

Fish were randomly divided into groups, and a hole was made in their skull bilaterally. Groups of fish received no injection, microinjection of saline, or microinjection of $10 \mu \mathrm{g} / \mu \mathrm{l}$ of carboxy-PTIO to their telencephalon immediately following training sessions on Experimental Days 1, 4, and 8. On Experimental Day 11, fish were tested without injection.

\section{Results}

Because there were no differences in avoidance responses during testing between no-injection and saline-injected fish, those fish were combined into one control group. A $t$ test on the percentages of avoidance responses during testing indicated a significant difference between the control fish and fish that fish received carboxyPTIO immediately following trainings. Fish that received carboxy-PTIO displayed significantly fewer avoidance responses during testing compared to the control fish. The fish that received carboxy-PTIO showed a mean of $12.5 \%$ avoidance responses, while the control fish showed a mean of $60 \%$ avoidance responses (Figure 1).

\section{Discussions \& Conclusions}

The control group was trained and tested using the same methods as the carboxy-PTIO fish, but received either no injections or injections with saline. There was no significant difference between fish that received no injec-

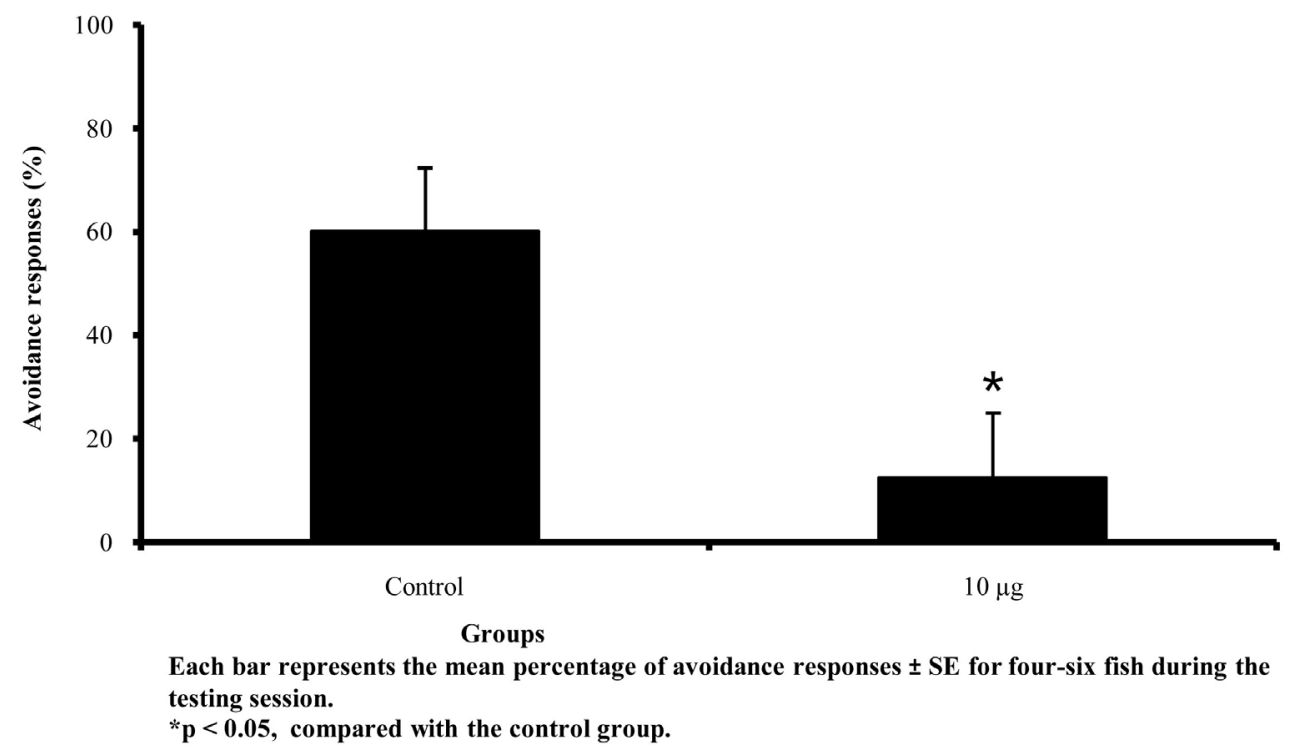

Figure 1. Post-training injection of carboxy-PTIO. 
tions and fish that received saline injections immediately following training. Therefore, neither the microinjection procedure itself nor the microinjection of saline to the goldfish telencephalon impaired active avoidance conditioning. Microinjections of carboxy-PTIO immediately following training impaired avoidance conditioning, suggesting that extracellular NO may be necessary for forming memory of avoidance conditioning in goldfish. The results of the current study confirm the finding that telencephalic NO is involved in memory consolidation [10]. Thus, our previous results with D-AP5 [9] and current results with carboxy-PTIO together suggest that the NMDA receptors are involved in learning or the process that is completed during training, whereas the NO is involved in memory consolidation or the process that is normally completed sometime following the learning experience.

\section{Acknowledgements}

This work was supported in part by a Grand Valley State University grant-in-aid.

\section{References}

[1] Gilbert, M.E. and Mack, C.M. (1990) The NMDA Antagonist, MK-801, Suppresses Long-Term Potentiation, Kindling, and Kindling-Induced Potentiation in the Perforant Path of the Unanethetized Rat. Brain Research, 519, 89-96. http://dx.doi.org/10.1016/0006-8993(90)90064-I

[2] Cotman, C.W., Monaghan, D.T. and Ganong, A.H. (1988) Excitatory Amino Acid Neurotransmission: NMDA Receptors and Hebb-Type Synaptic Plasticity. Annual Review of Neuroscience, 11, 61-80. http://dx.doi.org/10.1146/annurev.ne.11.030188.000425

[3] O’Dell, T.J., Hawkins, R.D., Kandel, E.R. and Arancio, O. (1991) Tests of the Roles of Two Diffusible Subtances in Long-Term Potentiation: Evidence for Nitric Oxide as a Possible Early Retrograde Messenger. Proceedings of the National Academy of Sciences of the United States of America, 88, 11285-11289. http://dx.doi.org/10.1073/pnas.88.24.11285

[4] Lu, Y.-F., Kandel, E.R. and Kawkins, R.D. (1999) Nitric Oxide Signaling Contributes to Late-Phase LTP and CREB Phosphorylation in the Hippocampus. Journal of Neuroscience, 19, 10250-10261.

[5] Bon, C.M. and Garthwaite, J. (2003) On the Role of Nitric Oxide in Hippocampal Long-Term Potentiation. The Journal of Neuroscience, 23, 1941-1948.

[6] Lynch, M.A., Errington, M.L., Clements, M.P., Bliss, T.V.P., Redini-Del Negro, C. and Laroche, S. (1990) Increases in Glutamate Release and Phosphoinositide Metabolism Associated with Long-Term Potentiation and Classical Conditioning. Progress in Brain Research, 83, 251-256. http://dx.doi.org/10.1016/S0079-6123(08)61254-6

[7] Xu, X., Russell, T., Bazner, J. and Hamilton, J. (2001) NMDA Receptor Antagonist AP5 and Nitric Oxide Synthase Inhibitor 7-NI Affect Different Phases of Learning and Memory in Goldfish. Brain Research, 889, 274-277. http://dx.doi.org/10.1016/S0006-8993(00)03216-9

[8] Xu, X., et al. (1998) Comparison of the Amnestic Effects of NMDA Receptor Antagonist MK-801 and Nitric Oxide Synthase Inhibitors: L-NAME and L-NOARG in Goldfish. Behavioral Neuroscience, 112, 892-899. http://dx.doi.org/10.1037/0735-7044.112.4.892

[9] Xu, X., Bazner, J., Qi, M., Johnson, E. and Freidhoff, R. (2003) The Role of Telencephalic NMDA Receptors in Avoidance Learning in Goldfish (Carassius auratus). Behavioral Neuroscience, 117, 548-554. http://dx.doi.org/10.1037/0735-7044.117.3.548

[10] Xu, X., Bently, J., Miller, T., et al. (2009) The Role of Telencephalic Nitric Oxide and cGMP in Avoidance Conditioning in Goldfish (Carassius auratus). Behavioral Neuroscience, 123, 614-623. http://dx.doi.org/10.1037/a0015243 